\title{
Transrectal Prostate Biopsy Inside Closed MRI Scanner with Remote Actuation, under Real-Time Image Guidance
}

\author{
Gabor Fichtinger ${ }^{1,2}$, Axel Krieger ${ }^{1}$, Robert C. Susil ${ }^{2,3}$, Attila Tanacs ${ }^{1}$, \\ Louis L. Whitcomb ${ }^{4}$, and Ergin Atalar ${ }^{2}$ \\ ${ }^{1}$ Engineering Research Center, Johns Hopkins University, Baltimore, MD, USA \\ gabor@cs.jhu.edu \\ ${ }^{2}$ Department of Radiology, Johns Hopkins University, Baltimore, MD, USA \\ ${ }^{3}$ Dept. of Biomedical Engineering, Johns Hopkins University, Baltimore, MD, USA \\ ${ }^{4}$ Department of Mechanical Engineering, Johns Hopkins Univ., Baltimore, MD, USA
}

\begin{abstract}
We present the proof-of-concept prototype of a prostate biopsy robot to be used inside a conventional high-field MRI scanner. A three degree-offreedom (DOF) mechanical device translates and rotates inside the rectum and enters a needle into the body, and steers the needle to a target point pre-selected by the user. The device is guided by real-time images from the scanner. Networked computers process the medical images and enable the clinician to control the motion of the mechanical device that is operated remotely from outside the imager. The system is also applicable to localized prostate therapy and also demonstrates potential in other intra-cavitary procedures.
\end{abstract}

\section{Introduction}

Prostate diseases represent a significant health problem in the United States. After cardiac diseases and lung cancer, metastatic prostate cancer is the third leading cause of death among the American men over fifty years, resulting in approximately 31,000 deaths annually. The definitive diagnostic method of prostate cancer is core needle biopsy. Annually in the U.S., approximately 1 million prostate biopsies are performed revealing around 200,000 new cases. Currently, transrectal ultrasound (TRUS) guided needle biopsy is the gold standard for the diagnosis of prostate cancer [1] and contemporary intraprostatic delivery of therapeutics is also primarily performed under TRUS guidance. This technique has been overwhelmingly popular due to its excellent specificity, real-time nature, low cost, and apparent simplicity. At the same time, however, TRUS-guided biopsy fails to correctly detect the presence of prostate cancer in approximately $20 \%$ of cases [2,3]. Also importantly, the transrectal ultrasound probe implies variable normal force on the prostate through the rectal wall, causing dynamically changing deformation and dislocation of the prostate and surrounding tissue during imaging and needle insertion, an issue that has to be eliminated in order to achieve accurate and predictable needle placement. MRI imaging has a high sensitivity for detecting prostate tumors $[3,4]$. Unfortunately, MR imaging alone, without concurrent biopsy, suffers from low diagnostic specificity. Therefore, our primary objective was to develop a prostate biopsy system that couples superior imaging quality with accurate delivery hardware, inside a conventional MRI scanner. The 
challenge was three-fold: (1) Conventional high-field MRI scanners apply wholebody magnets that surround the patient completely and do not allow access to the patients during imaging. The workspace inside the magnet is extremely limited, so conventional medical robots and mechanical linkages do not fit in the magnet. (2) Due to the strong magnetic field, ferromagnetic materials and electronic devices are not allowed to be in the magnet, which excludes the use of traditional electromechanical robots and mechanical linkages. (3) A real-time in-scanner guidance method is needed to operate the device.

Cormack, Tempany, Hata, D'Amico, et al. at the Brigham and Women's Hospital in Boston [5,6] proposed to use open MRI configuration in order to overcome spatial limitations of the scanner. This magnet configuration allows the physician to step inside the magnet and access the patient. The Brigham group proved the ability of MRI to detect cancer previously missed by TRUS biopsy and they performed successful targeted biopsy and brachytherapy inside the open MRI scanner. While this approach has opened up a new chapter in managing prostate cancer, the open MRI scanner limits its potentials. Compared to closed magnets, open magnets tend to have lower signal-to-noise ratio, so the images also tend to be of lower quality. The incurred cost and complexity of open MRI imaging are also substantial. The Brigham group applies transperineal access in biopsy, which it is significantly more invasive than the conventional transrectal approach.

Relevant work in robot assisted needle placement is also found in the literature. Recently, Fichtinger et al. [7] presented a 3-DOF remote center of motion robot for transperineal prostate access, the kinematic concept of which is not applicable to transrectal procedures. Rovetta et al. [8] applied an industrial robot to assist TRUSguided prostate biopsy with the use of a conventional end-shooting probe. The robot mimicked manual handling of TRUS biopsy device in the patient's rectum, in a telesurgery scenario. This system did not gain popularity, because the benefits of telesurgery did not compensate for the added cost and complexity of the robot and many problems of TRUS-guided free-hand biopsy still remained unaddressed. The Brigham group, Chinzei, Hata et al. [9] reported a robotic assistant for transperineal needle placement in open MRI. In this design, the MR-compatible motors are situated outside the high field zone and two manipulator arms reach into the scanner. The arms are encoded and tracked by a FlashPoint tracker simultaneously. Unfortunately, these geometric and kinematic concepts are incompatible with our application. Custom designed robots for use inside conventional closed MRI have also been investigated: Kaiser et al. [10] developed a system for breast biopsy and Masamune et al. published another one for brain surgery [11]. The kinematics and design concepts of these are also not applicable in transrectal prostate biopsy.

Multiple investigators, including our group at the Johns Hopkins University have studied intra-operative CT and X-ray guidance of surgical robots using passive fiducials attached to the interventional device. MRI imaging, however, is unique by offering the opportunity to apply micro-coil antennas as active fiducials [12]. In this scenario the signal processing software "listens" to a prominently present "signature" from the fiducial coils, allowing for true accurate real-time calculation of the coil positions, then using the these positions as rigid body markers on the device.

Our approach to image-guided prostate biopsy differs from other all the above solutions, in several aspects. Our objectives were to employ high resolution MRI imaging inside a closed MRI scanner, maintain the safe transrectal access, while replacing the manual technique with a remotely controlled needle insertion system, in order to 
maximize needle placement accuracy and also minimize dynamic tissue deformation. We also developed real-time tracking and guidance for the device, using solely the MRI scanner, without external tracking instruments.

\section{Materials and Methods}

\section{Systems Design}

The systems concept is shown in Figure 1. The device is secured to the table of the scanner with an adjustable mount that allows for flexible initial positioning. The patient is positioned comfortably on the scanner's couch in prone position with slightly elevated pelvis and the device is introduced to the rectum. A thin rigid sheath attached around a tubular obturator makes contact with the rectum, while the obturator can slide smoothly inside the sheath. The sheath prevents the obturator from causing mechanical distortion to the rectum wall and prostate while it is moving inside the rectum. After a satisfactory initial position is achieved, the mount is secured to hold this position. Using the sliding table of the scanner, the patient and device are moved into the scanner's magnet. The MRI scanner produces signal with the patient and device in the field, at the same time. Using signalprocessing tools, we

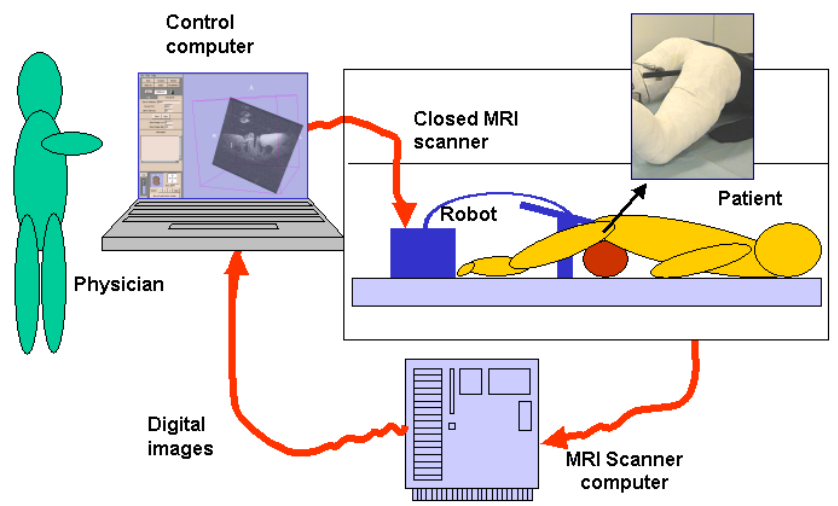

Figure 1: Schematic view of the system determine the spatial relationship between the device and the coordinate system of the MRI scanner. The images are transferred to a computer that produces a 3D representation of the device superimposed on the anatomic images. The physician interacts with the display and selects the target point for the needle. The computer calculates the kinematic sequence to bring the needle to the selected target position. The device realizes 3-DOF motion: translation of the end-effector inside the rectum, rotation of the end-effector around the axis of translation, and the depth of needle insertion. The order of translation and rotation are interchangeable, but both must be completed before the needle is inserted. The computer can also simulate the sequence of motion by moving a 3D model of the device, so that the physician could verify that the calculated sequence of motion would indeed take the needle from its current position to the pre-selected target position. The computer displays the three motion parameters to the operator. In the current embodiment of the system, the motion stages are powered manually, because motorized actuation was not considered to be a critical element in our proof of feasibility prototype. While the actuation of the device is in progress, the MRI scanner is collecting images in continuous mode and sends them immediately to treatment monitoring computer. The computer processes the image data and 


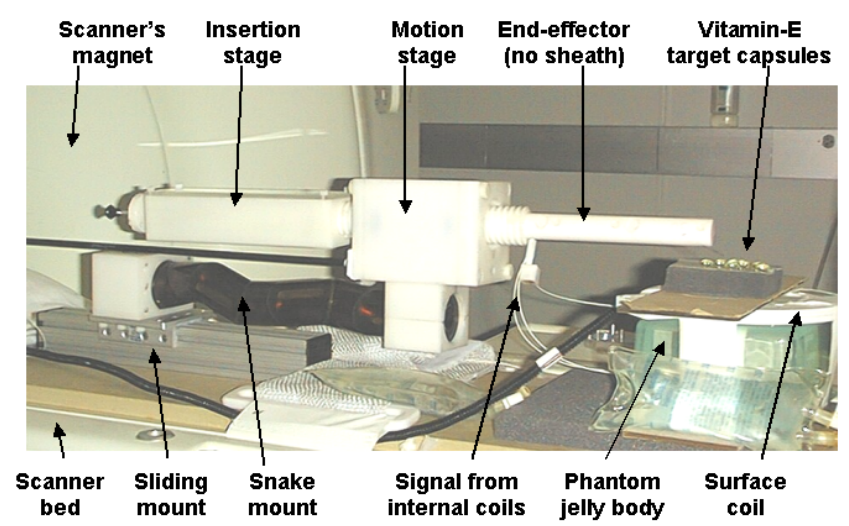

Figure 2: Experimental setup visualizes the current image, with the model of the device superimposed in the scene, allowing the physician to monitor the motion of the device toward its target. The three parameters of motion (translation, rotation, insertion depth) are recalculated in each imaging cycle, enabling real-time dynamic control of the device. When the mo-

tion is motorized, we will have achieved the ideal of "point-and-click" surgery, when the surgeon points and clicks on a target in a computer screen, then a robot moves the needle and inserts it into the target, under real-time imaging surveillance but without manual intervention. An experimental setup with phantom is shown in Figure 2.

\section{Description of the Device}

The assembly of the needle insertion device is shown in Figure 3. The device consists of the following main components: (1) end-effector that is introduced into the patient's rectum, (2) motion stage to provide translation and rotation for the

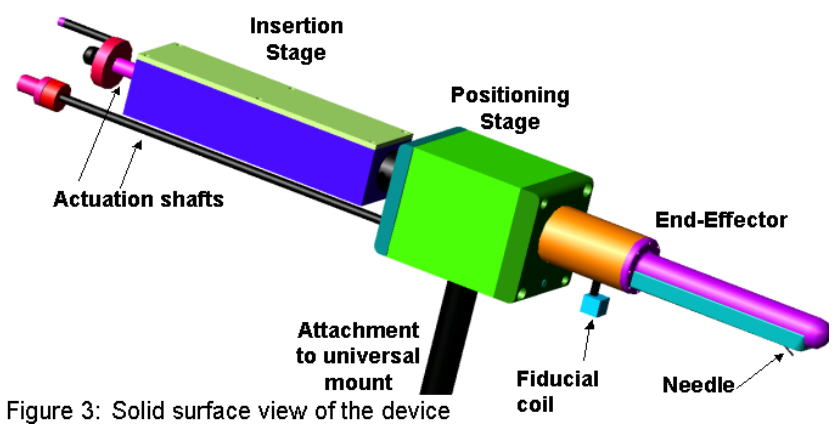
end-effector, (3) in-

sertion stage to enter the needle into the prostate and retract it, (4) adjustable mount to bring and secure device in optimal initial position, (5) actuation shafts for remote operation from outside the scanner. In order to maintain material compatibility with MRI, the device was manufactured entirely from plastic, except the antennas, the needle, and two aluminum parts: the trigger of the biopsy needle and a sliding rail under the universal mount, but these two are situated far away from the field of imaging and produce no measurable artifact.

The end-effector is composed of a tubular obturator that translates and rotates in a thin rigid sheath. The sheath minimizes the deformation and displacement of the organ during positioning of the probe and it maintains a stationary position in reference to the organ of interest. The sheath also contains an MRI imaging loop antenna that produces real-time anatomic images stationary with respect to the subject anatomy. A small window is cut on the sheath through which the needle enters the body. A curved 
guiding channel is formed inside an obturator for a flexible standard MRI-compatible 18G biopsy needle. The needle exits on the side of the obturator and enters into the body through the rectum wall. Upon exiting the channel, the needle follows a straight trajectory. The obturator also holds three registration coils as active fiducials providing the spatial position of the probe in the MRI coordinate system.

The positioning stage transforms rotation of two concentric shafts into translation and rotation of a main shaft that serves as transmission to the end-effector. The concentric shafts are actuated from outside the scanner. The needle insertion stage transforms rotation of a knob into a well-defined insertion of the needle to a pre-determined target depth and actuates the shooting mechanism of a biopsy gun. In the current prototype, we adapted an 18G standard prostate biopsy needle (Daum GmbH, Schwerin, Germany). The insertion stage can also rotate the needle while it is being translated, thus achieving straight needle path when the needle needs to come out in a steeper angle from the end-effector.

\section{Image Guidance}

We apply computational image guidance, based on real-time collected MRI images. In this scenario, fiducial markers in a known geometric distribution are rigidly incorporated with the end-effector. Images are acquired with the device and patient together in the field of view. We use active MRI imaging antennas instead of traditional passive fiducial markers. Three imaging coils are situated in the end-effector of the device. Each coil winds around a small capsule containing gadolinium solvent, in order to provide strong signal in the vicinity of the coil. Two coils are located in the central axis of the endeffector to encode trans-
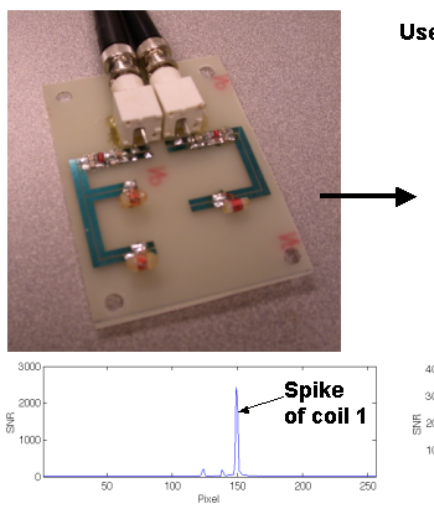

Signal on channel 1
Use the calculated coil positions as rigid body fiducials
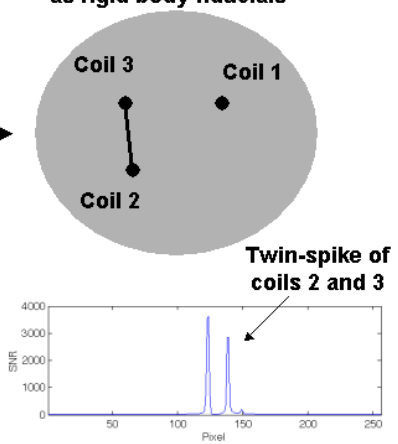

Signal on channel 2

Figure 4: Dynamic Tracking Method

lation and the third coil is located off-axis to encode rotation. (More coils could provide redundancy to increase accuracy, but would also leave less channel capacity for anatomical imaging.) Figure 4 shows an early prototype of the tracking hardware. Each of these coils produces a single spike in the MR signal. Unlike other imaging modalities, MR gives us the ability to take direct, 1D projections. We acquire ten 1D projections of these fiducials along different orientations. This yields an overdetermined linear system for the positions of the points, which we can then solve for the coil locations. This yields enough information to define the scan plane. All together, the tracking sequence takes $50 \mathrm{~ms}, 5 \mathrm{~ms}$ for each 1D projection. 


\section{Calibration}

The registration coils were fabricated manually, so the location of their signal centers with respect to the end-effector cannot be not be known without prior calibration. This information is constant for the entire lifetime of the device, assuming always the same image acquisition and processing parameters. We apply two tubes filled with gadolinium solvent. The first tube is placed inside the end-effector, while the other was attached around the needle. In a volumetric image of the device, the two tubes fully define the end-effector, while we also have readings on the coil positions. We repeat the calibration several times, in order to reduce the effects of measurement errors by simple averaging.

\section{Results}

The mechanical components of the device were analyzed separately, in laboratory environment. The accuracies of the translational and rotational mechanisms were found to be $1 / 100 \mathrm{~mm}$, while the accuracy of the needle insertion stage is 0.1 $\mathrm{mm}$. The computer software developed for calculation of targeting sequence, inverse and forward kinematics was tested on synthetic and real measurement and was found to be significantly more accurate than the mechanical com-

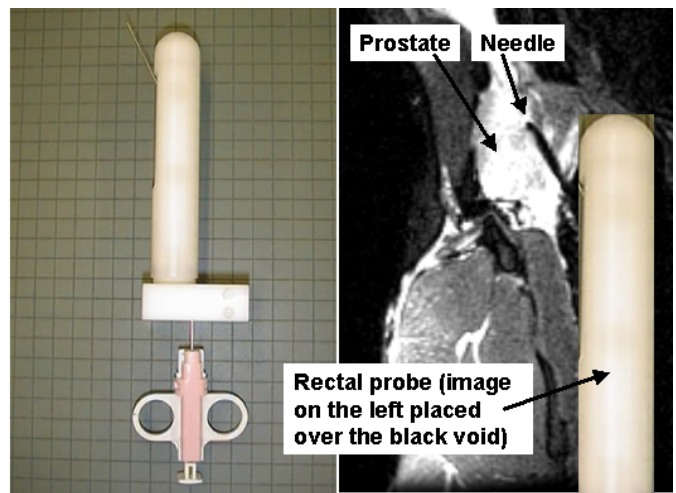

Figure 5: End-effector in live canine ponents themselves. The application and anatomical; feasibility of the end-effector was tested in vivo, when we performed transrectal needle biopsy of the prostate in a dog. Figure 5 shows the rectal sheath (left) and an $18 \mathrm{G}$ biopsy needle entered through this end-effector (right). These experiments revealed that the combined system error has only one significant component and that originates from signal processing. The premise of our registration technique is that the readings on the positions of the fiducial coils define a rigid triangle at all times. We have performed extensive experiments that proved the feasibility of this concept. We used a mechanical frame that allowed for controlled translations and rotations of a board holding three fiducial coils in the same constellation as they are built in the end-effector. The three coils always define a triangle. We calculated the difference between the expected and measured centroids of the triangle and the difference between the expected and measured plane normal vectors of the triangle, which were $0.41 \mathrm{~mm}$ and 1.1 degree, respectively. Experiments were directed toward determining the effect of these errors on actual targeting accuracy. Total systemic accuracy has been tested in phantoms (Figure 6). Needle placement was found to be consistent throughout the field of imaging. The accuracy of needle placement was determined visually and it was below $2 \mathrm{~mm}$ in all trials. We expect to achieve similar 
accuracy in in-vivo, however, effects of possible displacement of the prostate during insertion is an important factor to be explored.

\section{Conclusion}

We successfully demonstrated a proof-of-concept prototype for in-MRI biopsy of the

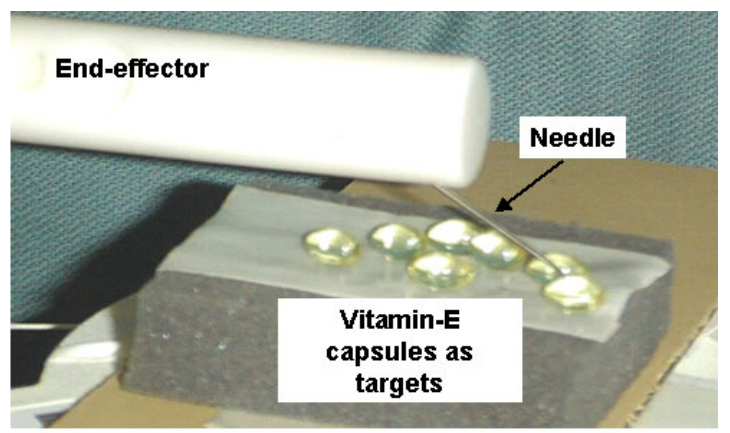

Figure 6: Needle hitting a target in phantom experiment prostate, under real-time image guidance, with remote actuation. We performed phantom and initial in-vivo canine studies. Determination of the combined systemic needle placement accuracy (considering errors from calibration, imaging, signal processing, target calculation, and mechanical actuation) is a work in progress. Our results so far justify the development of an advanced prototype suitable for human trials.

\section{Acknowledgements}

The authors acknowledge the support of the National Science Foundation under the Engineering Research Center grant \#EEC-9731478. We are grateful to Theodore DeWeese, MD (Johns Hopkins University) for his guidance in clinical matters and to Dan Stoianovici, $\mathrm{PhD}$ (Johns Hopkins University) for contributing the universal snake mount (Figure 2) to our experiments.

\section{References}

1. Presti JC Jr. Prostate cancer: assessment of risk using digital rectal examination, tumor grade, prostate-specific antigen, and systematic biopsy. Radiol Clin North Am. 2000 Jan;38(1):49-58. Review

2. Norberg M, Egevad L, Holmberg L, Sparen P, Norlen BJ, Busch C. The sextant protocol for ultrasound-guided core biopsies of the prostate underestimates the presence of cancer. Urology. 1997 Oct;50(4):562-6.

3. Wefer AE, Hricak H, Vigneron DB, Coakley FV, Lu Y, Wefer J, Mueller-Lisse U, Carroll PR, Kurhanewicz J. Sextant localization of prostate cancer: comparison of sextant biopsy, magnetic resonance imaging and magnetic resonance spectroscopic imaging with step section histology. J Urol. 2000 Aug;164(2):400-4.

4. Yu KK, Hricak H. Imaging prostate cancer. Radiol Clin North Am. 2000 Jan;38(1):59-85, viii. Review.

5. Cormack RA, D'Amico AV, Hata N, Silverman S, Weinstein M, Tempany CM. Feasibility of transperineal prostate biopsy under interventional magnetic resonance guidance. Urology. 2000 Oct 1;56(4):663-4. 
6. D’Amico AV, Tempany CM, Cormack R, Hata N, Jinzaki M, Tuncali K, Weinstein M, Richie JP. Transperineal magnetic resonance image guided prostate biopsy. J Urol. 2000 Aug;164(2):385-7.

7. G. Fichtinger, T. L DeWeese, A. Patriciu, A. Tanacs, D. Mazilu, J. H. Anderson, K. Masamune, R H. Taylor, D. Stoianovici: Robotically Assisted Prostate Biopsy And Therapy With Intra-Operative CT Guidance: Journal of Academic Radiology, Vol 9, No 1, pp. 6074

8. Rovetta A, Sala R: Execution of robot-assisted biopsies within the clinical context., Journal of Image Guided Surgery. 1995;1(5):280-287

9. Chinzei K, Hata N, Jolesz FA, Kikinis R, MR Compatible Surgical Robot: System Integration and Preliminary feasibility study, Medical Image Computing and Computer-assisted Intervention 2000, Pittsburgh, PA. Lecture Notes in Computer Science, MICCAI 2000, Springer-Verlag, Vol. 1935, pp. 921-930

10. Kaiser WA, Fischer H, Vagner J, Selig M. Robotic system for biopsy and therapy of breast lesions in a high-field whole-body magnetic resonance tomography unit. Invest Radiol. 2000 Aug;35(8):513-9.

11. Masamune et. al., Development of an MRI-compatible needle insertion manipulator for stereotactic neurosurgery. Journal of Image Guided Surgery, 1995, 1 (4), pp. 242-248

12. Derbyshire JA, Wright GA, Henkelman RM, Hinks RS. Dynamic scan-plane tracking using MR position monitoring. J Magn Reson Imaging. 1998 Jul-Aug;8(4):924-32. 